\title{
A New Biophysical Analysis of the Twin Paradox: The Interaction between Velocity and the Variable Biological Clock
}

\author{
Fred H Thaheld* \\ Independent Researcher, USA
}

\begin{abstract}
Initial analysis of the twin paradox from a biophysical standpoint appears to reveal a new additional consideration that has been overlooked for over a century. That the traveling twin may come back even younger than has previously been considered due to his variable daily metabolic cycle or variable biological clock, with special emphasis on his period of sleep involving his normally slowed biological clock, becoming minutely further slowed during each such cycle, due to velocity continuing to interact with this reduced base rate and in accordance with the Lorentz factor. It is well known that time changes for anything moving, but in addition humans, along with a vast range of other animate entities, have their own time or biological clock naturally changing in a daily cyclical fashion from a higher to a lower rate encompassed by sleeping, which rate continues to be subject to increased time changes engendered by velocity. The twin on earth is subject only to constant gravity at $1 \mathrm{~g}$ and not to velocity. Further analysis reveals several problems with part of Einstein's original thought experiment, recently brought to light in results released by NASA regarding the impact upon a twin's physical condition after being in a hypogravity low earth orbit for almost a year. In addition, that one can now distinguish between falling in a gravitational field and floating in space, since it has been revealed that a floating astronaut is subject to numerous discernible biophysical changes while the falling person is not, casting doubt on the validity of the weak principle of equivalence.
\end{abstract}

\section{Keywords}

Aging, entropy, Hypogravity, Hypergravity, Lorentz factor, Principle of equivalence, Twin paradox, Variable biological clock, Velocity, Zero gravity

\section{Introduction}

The twin paradox is a thought experiment based upon Einstein's paper on special relativity in 1905 [1]. In there he dealt with 2 clocks that were brought together, synchronized and then one was moved away and returned while the other remained in the same place on earth. Upon return the traveling clock would be found to be lagging behind the nontraveling clock. He later modified this approach so that the clocks were replaced by a living organism and this was further modified by Langevin so that the living organism is a man who remains on earth and the traveling one is his twin [2]. In this instance Langevin was utilizing the Lorentz factor or term by which time, length and relativistic mass change for that object while it is moving.

It has been noted many times in the literature that if one twin (Alice) stays on earth while the other twin (Bob) proceeds out into space at a high velocity as represented by the Lorentz factor, and then returns after a period of time, that Bob returns much younger than the stationary twin. The degree of his youth depending upon the length of the period of velocity and its proximity to the speed of $c$ which he undergoes during his trip. He is said to be in 2 different inertial frames during his round trip, while the twin on earth is in the same inertial or rest frame, not subject to the effects of velocity. This reasoning has been shown to be correct in experiments conducted with atomic clocks flown in aircraft and satellites [3], and in particle accelerators by measuring the time dilation of circulating particle beams [4]. This all takes place in accordance with the Lorentz factor.

As will be shown, this thought experiment regarding the twin paradox (which is really not a paradox) [5], may not be completely correct from an animate or biophysical standpoint, when one more closely examines the reduced bodily functions of the traveling twin within the framework of his naturally occurring variable biological 'clock', during that 8 hour portion of his 24 hour daily cycle while he is normally sleeping, in relation to the velocity which he continues to

*Corresponding author: Fred H Thaheld, Independent Researcher, USA

Accepted: August 08, 2019

Published online: August 10, 2019

Citation: Thaheld FH (2019) A New Biophysical Analysis of the Twin Paradox: The Interaction between Velocity and the Variable Biological Clock. Insights Biomed Res 3(1):56-61 
experience while sleeping at this reduced base rate. The variable biological clock is a complex mixture or summation of a vast range of interacting and contributing microscopic and macroscopic components possessing their own clock rates, with the visible and measurable end results showing up as changes in respiration, heartbeat, temperature, blood pressure, EKG and EEG readings among other elements, representative of tensed time or past, present and future.

It is not the purpose of this analysis to propose a different approach to the thought experiment of Einstein involving special relativity, but to possibly add an additional element which appears to have gone unexplored to date. Also, to address a serious problem with the twin paradox as it relates to recent findings regarding the deleterious influence of either hypogravity, hypergravity or zero gravity on human and other animate physiology in addition to those hazards posed by space radiation. In addition, to now question the validity of the weak principle of equivalence based on these findings.

\section{The Interaction between Velocity and the Variable Biological Clock with Emphasis on the Sleep Cycle}

It is well known that human subjects usually sleep for a duration of $\sim 8$ hours/day or $1 / 3^{\text {rd }}$ of the total time available to them during the 24 hour cycle here on earth which is referred to as the circadian rhythm. During this period of time while sleeping, bodily functions can slow down, with variations as to the length, depth and periodicity of these changes $[6,7]$. So, going from waking to sleeping represents one variable, with the events taking place while sleeping itself having their own variables as covered in the 5 stages of sleep. The heart rate can go down by from 10-30 beats per minute and breathing slows down with a reduction in the metabolic rate. While the overall process is not that simple, since there are 5 stages of sleep and there are periods when bodily activity can go up or down, for the purposes of this paper we will assume an overall average $25 \%$ metabolic reduction rate or slowdown of the variable biological clock during the 8 hours of sleep.

The traveling twin's body, while he is accelerating or at a velocity in an inertial frame, is subject to constantly slowly increasing effects, i.e. time for any moving object changes, so that when he goes to sleep and naturally slows down, because he is being subjected to this constant velocity while he is asleep, his variable biological clock will slow down even further, i.e. he will age slightly less than if he had remained awake during this cycle, with this effect happening ever so minutely per each 'daily' cycle, depending upon the Lorentz factor. This means that when he wakes up and returns to a minutely slower biological clock rate than if he had remained awake while being subjected to velocity, that he has ever so minutely aged less, and that this new minutely lower biological clock rate is still subject to velocity, with this 'dual' effect increasing minutely through each daily cycle of waking and sleeping. The effect will be very small in the initial stages of velocity as related to the speed of $\mathrm{c}$, but will gradually increase as per the Lorentz factor as the velocity increases. Because of this effect it means that the traveling twin will return even younger than people have calculated before since, for all of the roundtrip while he is in either one of his inertial frames, he will be sleeping approximately $1 / 3^{\text {rd }}$ of the time at even greater reduced variable biological clock levels, especially at very high velocities approaching c. If he were able to remain asleep for the entire trip or be placed in a hypothermic state he would accordingly come back even younger than if he had stayed awake.

Now, let us consider the condition of the twin on earth in light of the above reasoning. Since he will also be going through a 24 hour daily cycle, of which 8 hours will consist of sleeping, he is only subject to the constant inertial frame's normal gravitational effect upon his variable biological clock and is thus not subject to any of the additional time changes resulting from velocity, since he is not moving as far as the Lorentz factor is concerned, and will age at the normal rate whether awake or sleeping.

\section{Additional Considerations Involving the Twin Paradox and Derivative Issues}

When we first started writing this paper on a possible relationship between the variable biological clock, velocity and the twin paradox, we did not realize at the time that in the course of doing so we would automatically be led into briefly exploring related issues such as gravitational biology and the influence of hypogravity, hypergravity and zero gravity, the biological arrow of time, tensed and tenseless time, entropy, the measurement problem and the question of the equivalence of falling vs. floating. At this time (no pun intended!) we would like to proceed a little further in this new direction and see where it might lead us.

\section{Gravity and Its Effect on Life on Earth and in Space}

One critical factor in the creation of life and its evolution since life first began on earth is the force of gravity, which has remained essentially constant at $1 \mathrm{~g}$ since the formation of this planet [8]. All biological processes, whether plant or animal are accustomed to this ever present force and have evolved to rely upon and cope with it [9]. Even small variations in gravity of either a hypogravity or hypergravity nature can have a significant impact on the health, function and system of organisms. The size of single biological cells is inversely proportional to the strength of the gravitational field exerted on the cell. That is, in stronger gravitational fields the size of cells decreases and in weaker gravitational fields the size of cells increases. Gravity is thus a limiting factor in the growth of individual cells. This is somewhat surprising since in the case of gravity we have been taught that it is the weakest of all the 4 fundamental forces, which includes the electromagnetic, weak and strong interactions, operating as discrete quantum fields at extremely short microscopic or quantum distances in the inanimate realm in the case of the latter 2 forces. Although gravity is some $10^{40}$ weaker, its full force or power is felt at macroscopic distances. And yet a small variation in what is already a weak force exerts a major influence at the animate cellular quantum level due to its nature as 
an omnipresent and enveloping or immersive field, with its space-time implications at the microscopic cellular level and its ability to transmit its effect at the speed of $c$.

As a striking example in the case of spaceflight, experiments have shown that alterations in metabolism, immune cell function, cell division and cell attachment can occur in the microgravity $\left(<10^{-3} \mathrm{~g}\right)$ of space [10]. For instance, after several days in microgravity human immune cells were unable to differentiate into mature cells. It has also been shown that mammary metabolic activity in pregnant rats is significantly increased in response to space flight [11]. Understanding gene or protein expression using proteomic or genomic approaches is the key to unfolding the mechanisms behind hypogravity-induced problems which are involved in the alteration of cellular functions such as proliferation, differentiation, maturation and cell survival in areas such as bone cells, muscle cells, immune system cells and nervous system cells of astronauts $[12,13]$. This is in addition to changes in brain volumes, mainly decreased cortical volume and increased CSF subarachnoid and ventricular volume.

In the case of hypergravity, where mammals were subjected to different rates of increased gravity in the ranges of $1.5,1.75$ or $2.0 \mathrm{~g}$ through the use of a centrifuge, the data demonstrates a remarkable continuum of response across the hypogravity and hypergravity environments, and support the concept that gravity is an important environmental cue that has shaped biological responses within a very narrow range going back to the very beginning of life [9]. It is the only element in the complex environment that has remained constant and unchanged both before and after the first cellular life appeared. One could almost be justified in saying that we are looking at another example of fine tuning in the universe, but we should probably wait until either the Mar's trip or the Search for Extraterrestrial Intelligence (SETI) begins to bear fruit, and we can then hopefully see what the cellular animate results turn out to be based upon various gravitational fields that differ from ours.

\section{The Case of the Stationary Observer and 3 Clocks}

As is well known in special relativity a stationary observer watching an astronaut speeding by will observe his clock running slower than the observer's clock. This however is not totally correct for a moving variable biological clock as represented by the traveling twin, which can either slow down or speed up on a regular cyclical basis on its own at specific intervals relative to a stationary observer. So, at various times a stationary observer looking at the traveling twin's variable biological clock as represented by the heart rate or respiration, might see it going slower and at other times faster in contrast to the traveling twin's normal clock which is always running slower in comparison to the stationary observer's clock. However, since the twin is always moving at velocity, time would still be basically changing or slowing down overall, i.e. aging would continue to be slowed whether the variable biological clock is going faster or slower as per each cycle depending upon which part of the cycle one is observing.

\section{The Traveling Twin's Limitation as to Length of Trip and Velocity}

It now appears that the traveling twin will be restricted as to both the length of his trip and the period of time at velocity due to serious health considerations which were previously cited and which have heretofore not been addressed with respect to the twin paradox. Let us assume that a spaceship at departure commences accelerating at $1 \mathrm{~g}$ or $9 \mathrm{~m} / \mathrm{s}^{2}$. In close to a year it would be moving at a velocity $v=0.8 \mathrm{c}$ or $80 \%$ of the speed of light. Since the twin is moving at a high velocity, time is changing and he will therefore be aging at a much slower rate than his twin back on earth in accordance with special relativity. However, when he stops accelerating at $1 \mathrm{~g}$ and velocity alone comes into play, time will still be changing for him but, he will commence floating in space, just like the astronauts in the space station, and subject to the multiple deleterious effects of zero gravity $[13,14]$. When the identical twin in the recent NASA experiment returned to earth, he did come back extremely minutely younger than his twin on earth by about 13 microseconds, due to the velocity of his ship at $17,000 \mathrm{mph}$ for a period of 11 months. While significant changes were observed in association with the period of the spaceflight, the majority of these returned to their preflight state within the time period of the study [13]. These included changes in telomere length, gene regulation, carotid artery dimensions, subfoveal choroidal thickness and serum metabolites, among others. Some additional factors were significantly affected by the stress resulting from the return to earth including cognitive performance, immune response gene networks and inflammation cytokines. However, persistent changes were observed for a few measures even after a period of over 6 months back on earth such as increased numbers of short telomeres, some genes' expression levels, attenuated cognitive function and increased DNA damage from chromosomal inversions [13]. This does not include other unknown problems waiting to make their appearance or yet to be discovered especially in the area of genetics. While NASA's study is limited for statistical purposes due to there being just 2 related participants, and not enough time has passed to completely eliminate potential problem areas, numerous studies going back over 50 years with over 550 unrelated astronauts reveal a whole host of health problems of a short and long term nature ranging from bone and muscle deterioration, to balance disorders and changes in the immune system, some of which have only made their appearance in later years especially as regards cardiovascular disease $[13,14]$. One of the major problems is our lack of knowledge as to what the purpose still is of a wide range of genes and what happens when they are subjected to zero gravity like on a lengthy trip to Mars. The analogy which immediately sprang into the author's mind has to do with the unknown and long term health effects which were only gradually exposed over a period of years following the use of thalidomide, Agent Orange and fluorocarbons with regards to the Antarctic ozone hole. A future study of the health of offspring conceived after astronauts have been in microgravity or zero gravity for a period of time might prove to be of interest, even though of a limited statistical nature, especially as regards possible transmitted genetic impacts. 
What this means with regards to the twin paradox thought experiment is that the traveling twin will never be able to attain the full extent of the roundtrip as envisioned by Einstein and Lorentz, since when he proceeds from the acceleration phase at $1 \mathrm{~g}$ to the velocity phase alone at any point in his trip and commences floating in zero gravity, he will suddenly begin to face a range of health issues no matter what the Lorentz factor or velocity is. This has been verified by the recent NASA twin's study which was performed at an extremely low velocity, many orders of magnitude lower than any velocity envisioned in the twin paradox. If so much damage can be done at such a low velocity what will happen at higher velocities to be encountered in the twin paradox? Interestingly, once he commences floating, he will continue aging less at one biological level for a period of time due to velocity, while simultaneously aging more at another biological level due to zero gravity, resulting in floating in space! Time does change for the moving twin, but it has now become a 2-way street depending upon the context and in the end the animate entropy piper must be paid with an increase in disorder, much to the detriment of the astronaut's health.

And, even if NASA was to alternately develop a propulsion system capable of higher initial velocities for lengthy periods of time at even a low level of 2-3 $g$, the astronauts would then be faced with the problem of hypergravity and its effect upon their biological systems, just the same as if they were in a centrifuge. Furthermore, in the twin paradox since deceleration is also involved, if it was undertaken in excess of $1 \mathrm{~g}$, once again hypergravity with its health impacts would rear its head. There is thus a very narrow gravitational window between hypogravity and hypergravity where biological systems would be safe from harmful effects.

This is in addition to those effects arising from either regular cosmic rays, ultra high energy cosmic rays (UHECR) or rare extreme energy cosmic rays (EECR) which would have their own potential for causing serious damage. This is especially relevant at the genetic or DNA level where there are still so many unknowns, since the energy of any cosmic rays is magnified by many orders of magnitude depending upon the velocity of the traveling twin, and furthermore he will be outside the shielding provided by the earth's magnetic field [15].

\section{Gravity + Quantum Mechanics = Life + A Little Bit More?}

Based upon the above facts regarding the important role that gravity plays in the successful maintenance of life within such a narrow range of force, it is safe to conjecture that single celled life got its start due to gravity operating in close conjunction with quantum mechanics at the microscopic level. And, that without gravity, even with a super abundance of the necessary raw materials at the quantum level, the first vestiges of life would never have appeared and thrived. If gravity and quantum mechanics were operating in such close harmony to create the very first primitive life, does this point to some kind of natural unification of the two at this point in time as regards quantum gravity or auger well for its solution at some future date, especially within the animate realm?
And, that the initial cellular animate creation over $3.5 \times 10^{9}$ years ago also represents the first movement from an inanimate tenseless time associated with gravity and quantum mechanics, to an animate tensed time progressing from the microscopic to the macroscopic over a period of several billion years [16]. This first appearance of cellular life also gave automatic rise to the biological arrow of time which involved the $2^{\text {nd }}$ law of thermodynamics and entropy in this first open system which possessed or developed the paradoxical dual ability of a short term increase in entropy with a long term decrease in entropy. How could such a counterintuitive situation as this come about?

As regards the increase in entropy, even though the cell or membrane is exchanging nutrients, energy and waste products with the surrounding macroscopic environment in addition to a whole host of other items, it is faced with an increase in disorder and entropy as a result of aging, and eventual death. However, it is able to pass on its genetic components to the next generation with improvements over a long period of time, so that each time entropy increases as the end result of maximum disorder and death, the genetic components or DNA provide an offsetting temporary decrease in entropy through generations of offspring, guaranteeing an improving future. It is in these genetic components that we can see the past, present and future, which we are now able to manipulate in a hopefully beneficial manner with an increasingly more predictable future in mind. This leads to the postulation of a guided biological arrow of time equivalent to the concept of 'manifest destiny'. These improvements in the specific areas of intelligence, reasoning and creativity along with the usual unexpected dose of serendipitous events, now allow these selected sentient descendants of the original cellular life to modify their genetic components with improvements of their own, not only improving upon, but accelerating the natural progression of evolution in selected areas.

A specific example of this quickly evolving trend is a newly developed molecule known as CRISPR/CAS9 which can be designed to seek out and alter genes in plants and animals [17]. With its potential for gene targeting and genomic applications, any gene whose sequence is known can be turned off or on, replaced or removed. When it is eventually combined in the future with quantum computation and $\mathrm{Al}$ in a self-improving hybrid animate-inanimate form, we will get a long term directed decrease in entropy in the animate realm with us standing on the sidelines as monitoring observers! However, on the other side of the coin, this molecule CRISPR/ CAS9 comes with a major serious problem in that this whole gene-editing technology is so simple to use that it now allows a wide range of individuals, some with little technical training and little ethical constraints, to create all kinds of genetic mischief on a vast scale with potential dire implications for humanity.

\section{The Measurement Problem}

Now, how could this first cellular life have anything to do with the measurement problem? First you must visualize that the first cellular life or prokaryote, varying from about 0.1 to $5 \mu \mathrm{m}$ in diameter, has a very thin membrane or boundary ap- 
proximately $4 \mathrm{~nm}$ thick separating it from the outside macroscopic environment and, that without this membrane boundary, life could have never appeared. On the outside the environment provided the slurry of molecular nutrients and energy, of which only a select few were allowed or able to pass through the membrane to the inside of the cell or constantly impinged upon the membrane. On the inside the cell utilized the nutrients and the resulting waste products were passed through the membrane back to the outside while constantly impinging upon the inside. The ever present gravitational field, while exerting a minute downward force on the cell and its membrane, not only passed through the cell, but the cell was constantly immersed in it. One could then argue, as has been done in the past, that any of these items, including any motion by the cell or its membrane boundary relative to them or vice versa, could constitute a measurement whether of a nutrient, waste product, energy, electromagnetic or gravitational field nature, and that we could refer to all these disparate elements as information $[18,19]$. That this would always be instantaneous, continuous, simple and deterministic and, depending upon one's point of view, can represent either a natural collapse of the wave function or a natural and inherent part of cellular functioning, and would represent a $100 \%$ naturalization of the Born rule as has been elaborated upon before [20]. As you will recall John Bell raised an identical issue decades ago when he asked the question as to whether collapse of the wave function was performed by the first cellular life or whether it had to wait for a college professor to appear.

\section{Are Falling and Floating Equivalent in the Weak Principle of Equivalence?}

Einstein said that he first got his idea of the equivalence principle one day when it came to him that falling in earth's gravity would be the equivalent of floating in space while undergoing uniform motion, and that there would be no way to distinguish between the two if one was inside a closed container conducting only internal measurements. The author had raised the question in a previous paper about the validity of this reasoning in view of recent experiments on earth and in space showing the appearance of phosphenes or flashes of light in the retina of astronauts in space as a result of interaction with cosmic rays and no phosphenes for humans shielded on earth [21].

It now appears that a more valid argument can be made showing that falling and floating are not equivalent, based upon recent experiments previously outlined in this paper. These have revealed that a twin floating while in hypogravity, and especially an astronaut floating in zero gravity, suffer various detrimental health effects which the twin on earth does not. That once again it is shown that falling in the earth's gravitational field would not be the equivalent of floating in space and that one can now readily distinguish between the two effects.

\section{Conclusion}

When this analysis of the twin paradox was first commenced it was with the view of just looking at the time dif- ference which appeared to arise when one took into consideration the relationship between velocity and the variable biological clock of the traveling twin. This was in contrast to the fixed base or noncyclical rate of the normal clock subject to velocity and the variable biological clock of the twin on earth which, while it is of a cyclical nature, is very critically not subject to velocity. The point was then initially arrived at that the traveling twin would come back even younger than had previously been calculated because the factor of the sleep cycle with its reduced metabolic rate and its change of its own time and the action of velocity further changed the time. And, if the traveling twin could somehow arrange to sleep the whole trip or be in a hypothermic environment, he would come back even younger!

Further analysis revealed however that Einstein's thought experiment, as it is applied in the animate realm, faced several major problems regarding health issues based upon data acquired both during and after a number of astronauts' flights both in low earth orbit and on the Apollo mission, with special emphasis on the recent and more relevant spaceflight involving the Kelly twins, with this taking place in a hypogravity state. Additional data derived from centrifuge experiments conducted under hypergravity conditions beyond $1 \mathrm{~g}$ with various mammals and cells, revealed further health problems which would also have a bearing upon the length and/or velocity of the twin's trip.

It is now beginning to appear that no matter what velocity the twin is proceeding at as per the Lorentz factor or the length of the roundtrip, that it will be a dangerous trip unless it is constrained by $1 \mathrm{~g}$ gravity as regards not only the time period of velocity, but the period of time which has to also be devoted to acceleration and deceleration in this entire process. And, unfortunately it also now appears that the initial analysis involving the variable biological clock will quickly crash upon the same gravitational shoals!

The argument was advanced that since gravity was playing such a dominant role in the creation and evolution of cellular and more complicated life, that it had to play this role especially in the initial stages, in such close conjunction with quantum mechanics that we would be justified in saying that we are looking at the possibility of animate quantum gravity. And, that while a number of experiments are now being proposed or conducted in the inanimate realm searching for quantum effects in gravity, it may have already been accidently observed in limited experiments in the animate realm which are now waiting on replication attempts under more stringent experimental conditions and, more critically, interpretation of the results obtained [22,23]. This reasoning is based on apparent continuous controllable nonlocal correlations between 2 separated but entangled macroscopic neuronal masses, mounted on microelectrode arrays, each possessing their own minute gravitational field [23]. This type of entanglement has also apparently been shown in the correlations in depolarizations between separated cloned neuronal cell cultures, which are awaiting additional investigation and verification [24].

And, while it is outside the main thrust of this paper, 
since the temperature in the spacecraft and that of the twin would also relate to the variable biological clock and have an influence upon the aging process in conjunction with velocity, this leads us to future consideration on the possibility of temperature acting as a quasi Lorentz factor, especially for living organisms, when it is reduced in an appropriate manner. I.e., whether $t$ could approximately equal $v$ ? This would mean that time could also change for any animate entity without the need for velocity, including being brought to a complete stop and, that if need be, temperature could also be combined with velocity.

\section{References}

1. Einstein A (1905) On the electrodynamics of moving bodies. Annalen der Physik 10: 891-921.

2. Langevin P (1911) The evolution of space and time. Scienta 10: 31-54.

3. Hafele JC, Keating RE (1972) Around the world atomic clocks: Observed relativistic time gains. Science 177: 168-170.

4. Bailey J, Borer K, Combley F, et al. (1977) Measurements of relativistic time dilatation for positive and negative muons in a circular orbit. Nature 268: 301-305.

5. Pesic $P$ (2003) Einstein and the twin paradox. Eur J Phys 24: 585590.

6. Carley DW, Farabi S (2016) Physiology of Sleep. Diabetes Spectr 29: 5-9.

7. Sleep (2019) Wikipedia.

8. Ross MD (1984) The influence of gravity on structure and function of animals. Adv Space Res 4: 305-314.

9. Chyba CF, Hand KP (2005) Astrobiology: The study of the living Universe. Annu Rev Astro 43: 31-74.

10. Effect of spaceflight on the human body (2019) Wikipedia.

11. Plaut K, Maple RL, Wade CE, et al. (2003) Effects of hypergravity in mammary metabolic function: Gravity acts as a continuum. J Appl Physiol 95: 2350-2354.
12. Nichols HL, Zhang N, Wen $X$ (2006) Proteomics and genomics of microgravity. Physical Genomics 26: 163-171.

13. Garrett-Bakelman FE, Darshi M, Green SJ, et al. (2019) The NASA twins study: A multidimensional analysis of a year-long human spaceflight. Science 364: 1-20.

14. Delp MD, Charvat JM, Limoli CL, et al. (2016) Apollo lunar astronauts show higher cardiovascular disease mortality: Possible deep space radiation effects on the vascular endothelium. Sci Rep 6: 29901-29910.

15. Chancellor JC, Scott BIG, Sutton JP (2014) Space radiation: The number one risk to astronaut health beyond low earth orbit. Life 4: 491-510.

16. Matsuno K (2018) Temporality naturalized. Philosophies 3: 45.

17. Jinek M, Chylinski K, Fondara I, et al. (2012) A programmable dual-RNA-guided DNA endonuclease in adaptive bacterial immunity. Science 337: 816-821.

18. Smith CJ (1975) Problems with entropy in biology. Biosystems 7: 259-265.

19. Matsuno K, Paton RC (2000) Is there a biology of a quantum information? Biosystems 55: 39-46.

20. Thaheld FH (2015) Can the Stark-Einstein law resolve the measurement problem from an animate perspective? Biosystems 135: 50-54.

21. Thaheld FH (2009) Can biophysics tell us something about the weak equivalence principle vis a vis the thought experiment of Einstein involving human subjects? Biosystems 97: 103-107.

22. Thaheld FH (2005) An interdisciplinary approach to certain fundamental issues in the fields of physics and biology: Towards a unified theory. Biosystems 80: 41-56.

23. Pizzi R, Fantasia A, Gelain F, et al. (2004) Nonlocal correlations between separated neural networks. SPIE 5436: 107-117.

24. Mihelic FM (2019) Experimental evidence supportive of the quantum DNA model. SPIE 10984.

DOI: $10.36959 / 584 / 447$

Copyright: (c) 2019 Thaheld FH. This is an open-access article distributed under the terms of the Creative Commons Attribution License, which permits unrestricted use, distribution, and reproduction in any medium, provided the original author and source are credited. 